\title{
ELECTRON TEMPERATURE IN SEMI-INSULATING GaAs FOR LOW ELECTRIC FIELDS
}

\author{
A. Zduniak, J. EUSaKowski \\ Institute of Experimental Physics, Warsaw University \\ Hoża 69, 00-681 Warszawa, Poland \\ AND G. NOWAK
}

Laboratory of Physics of Crystal Growth, Przyokopowa 28, 01-208 Warszawa, Poland

\begin{abstract}
Heating of electrons by electric fields smaller than that required for generation of domain oscillations was investigated in samples of EL2-rich semi-insulating GaAs. Current-voltage characteristics were measured as a function of temperature between $268 \mathrm{~K}$ and $330 \mathrm{~K}$. They exhibit a sublinear shape which is interpreted as a result of an enhanced electron capture on the EL2. The capture rate and the electron temperature as a function of the electric field was determined. A fitting procedure gave the value of electron capture cross-section on the EL2 to be $2.7 \times 10^{-13} \mathrm{~cm}^{2}$ which agrees with literature data.
\end{abstract}

PACS numbers: 72.20 . Ht

Long known spontaneous current oscillations in semi-insulating (SI) GaAs caused by high electric field domains propagation [1] have also been investigated during last years $[2,3]$. The mechanism responsible for this phenomenon is electric field enhanced capture of electrons from the conduction band on a deep centre. In particular, it is proposed that in undoped SI GaAs the level involved is the EL2 [4]. This idea was first put forward by Kaminska et al. [5] who explained the phenomenon as the following.

When the electric field $E$ applied to the sample is sufficiently high, the electrons in the conduction band gain enough energy to come over the configurational barrier which separates a free electron from its bound state on the EL2. This leads to a decrease in the free electron concentration and thus to a negative differential conductivity (NDC). The observed current oscillations are a result of drowning the system into the NDC regime.

This reasoning was recently put into a quantitative model by Johnson et al. [2] and Surma and Łusakowski [6]. The principal idea is to describe the electron gas by an electron temperature $T_{\mathrm{e}}$ which is different from the crystal lattice temperature $T$. The present paper deals with the range of electric fields below that 
required for the oscillations. An analysis of the experimental data allows to calculate $T_{\mathrm{e}}$ as a function of $E$ and shows that the capture rate of electrons from the conduction band on the EL2 increases with $E$.

All the experiments described below were performed on samples cut from one LEC wafer of undoped SI GaAs. A sample with six contacts was used for low field Hall measurements which gave the temperature dependence of the zero electric field mobility (which is constant for electric fields below $3 \mathrm{kV} / \mathrm{cm}$ [7]). Current-voltage $(I-V)$ characteristics were measured as a function of the temperature on another sample with two bar-shaped contacts (Fig. 1). The known

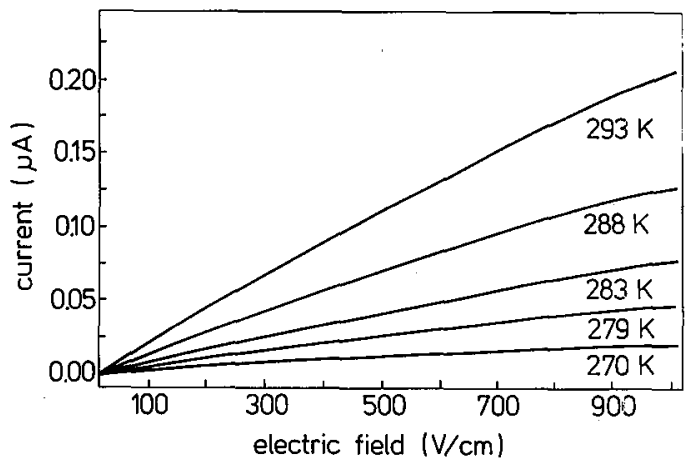

Fig. 1. Current-voltage characteristics for some of the temperatures.

value of the electron mobility allowed to calculate the concentration of free electrons $n$ as a function of the temperature and the electric field. Under the assumption that the occupancy of the EL2 changes only as a result of transitions to and from the conduction band, the condition of a stationary state takes the form $n c_{n}\left(N_{t}-n_{t}\right)=e_{n} n_{t}$, where $c_{n}$ is the capture rate, $e_{n}$ is the emission rate of the inverse process, $N_{t}$ and $n_{t}$ is the concentration of total and neutral EL2 centres, respectively. Since $n_{t}$ is given by the Fermi distribution, one gets $c_{n}=e_{n} /\left\{n \exp \left[\left(E_{t}-F\right) / k T\right]\right\}$, where $F$ is the Fermi level, $E_{t}=0.76 \mathrm{eV}$ is EL2 location below the conduction band and $k$ is the Boltzmann constant.

The crucial point in calculating $c_{n}$ is that $n$ is not assumed to be expressed by the formula $n=N_{\mathrm{c}} \exp \left[\left(F-E_{\mathrm{c}}\right) / k T\right]$, because one cannot describe by the equilibrial distribution the hot electron gas driven away from the equilibrium. Instead, we calculate $n$ from the $I-V$ curves. The value of $e_{n}$ is given by $e_{n}=$ $2.8 \times 10^{7} \times T^{2} \exp (-9450 / T) \mathrm{s}^{-1}[8]$ and is constant for electric fields less than about $100 \mathrm{kV} / \mathrm{cm}$ [9]. The Fermi level is calculated from the value of $n$ extrapolated to the zero electric field and is assumed not to depend on $E$, because the electric field causcs negligible changes in deep levels occupancy.

The results of the calculations for $T=288 \mathrm{~K}$ are shown in Fig. 2. $c_{n}$ is an increasing function of $E$ for all temperatures. The capture rate $c_{n}$ is given by the formula $c_{n}=v_{\text {th }} \sigma$, where $v_{\text {th }}$ is the thermal velocity of electrons and $\sigma$ is the capture cross-section. To describe the dependence of $c_{n}$ on the electric field we express $v_{\text {th }}$ and $\sigma$ by a field dependent electron temperature $T_{\mathrm{e}}$ : 


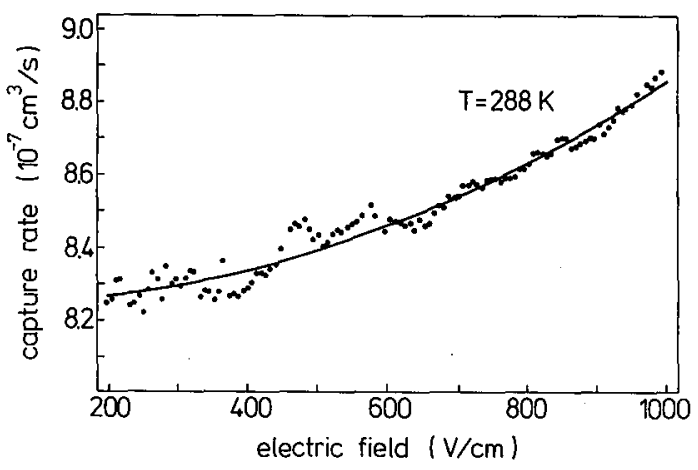

Fig. 2. An example of results of calculations of $c_{n}$ (dots) and the fitting procedure (full line) for $T=288 \mathrm{~K}$.

$v_{\text {th }}=\left(3 k T_{\mathrm{e}} / m^{*}\right)^{1 / 2}$ and $\sigma=\sigma_{\infty} \exp \left(-E_{\mathrm{b}} / k T\right)$, where $T_{\mathrm{e}}=T\left(1+\alpha E^{2}\right)[10], m^{*}$ is the free electron effective mass and $E_{\mathrm{b}}=0.063 \mathrm{eV}$ is the height of the configurational barrier of the EL2 [11]. In other words, we assume that the free electron distribution is Maxwellian, but characterized by $T_{\mathrm{e}}$ instead of $T$. We fit the $c_{n}$ on $E$ dependence using the above formulae taking $\sigma_{\infty}$ and $\alpha$ as the fitting parameters. We get $\sigma_{\infty}=2.7 \times 10^{-13} \mathrm{~cm}^{-2}$ independent of the temperature which agrees with literature data [12]. The value of $\alpha$ for different temperatures is scattered between $0.8 \times 10^{-5}$ and $1.3 \times 10^{-5} \mathrm{~K} \mathrm{~cm}^{2} / \mathrm{V}^{2}$. A mean value of $10^{-5} \mathrm{~K} \mathrm{~cm}^{2} / \mathrm{V}^{2}$ was used to calculate the ratio of energy relaxation time to mobility relaxation time [10]. We obtained the value of this ratio equal to about 150 which is the correct order of magnitude.

In conclusion, the proposed model is justified by the results which give the correct value of the EL2 cross-section. The results support also the model which connects the domain oscillations in SI GaAs with enhanced electron capture on the EL2.

\section{Acknowledgments}

The authors would like to thank Dr. E. Kamińska and Dr. A. Piotrowska for alloying the contacts to the samples and Prof. M. Grynberg for valuable discussions.

\section{References}

[1] D.C. Northrop, P.R. Thornton, K.E. Trezis, Solid State Electron. 7, 17 (1964).

[2] G.N. Maracas, D.A. Johnson, H. Goronkin, Appl. Phys. Lett. 46, 305 (1985).

[3] D.A. Johnson, R.A. Puechner, G.N. Maracas, J. Appl. Phys. 67, 300 (1989).

[4] M. Kamińska, Rev. Phys. Appl. 23, 793 (1988).

[5] M. Kamińska, J.M. Parsey, J. Lagowski, H.C. Gatos, Appl. Phys. Lett. 41, 989 (1982). 
[6] M. Surma, J. Łusakowski, unpublished.

[7] A.F. Volkov, Sh.M. Kogan, Usp. Fiz. Nauk 96, 633 (1968).

[8] H.C. Gatos, J. Lagowski, in: Proc. Mater. Res. Soc. Symp. Microscopic Identification Electronic Def. Semicond., San Francisco 1985, p. 153.

[9] J. Lagowski, D.G. Lin, H.C. Gatos, J.M. Parsey Jr., M. Kamińska, Appl. Phys. Lett. 45, 89 (1984).

[10] V. Boncz-Brujewicz, S. Kalashnikov, Fizyka pótprzewodników, PWN, Warszawa 1985.

[11] P. Dreszer, M. Baj, Acta Phys. Pol. A 73, 219 (1988).

[12] G.M. Martin, Electron. Lett. 13, 191 (1977). 